\title{
A review of the management of gait impairments in chronic unilateral upper motor neuron lesions
}

\author{
Jorik Nonnekes MD PhD ${ }^{1,2}$, Nathalie Benda MD PhD², Hanneke van Duijnhoven MD¹, \\ Frits Lem MD², Noël Keijsers PhD ${ }^{3}$, Jan Willem K. Louwerens MD PhD ${ }^{4}$, Allan Pieterse PT PhD ${ }^{1}$, \\ Bertjo Renzenbrink MD, ${ }^{5}$ Vivian Weerdesteyn PT PhD, ${ }^{1,3}$ Jaap Buurke PT PhD, ${ }^{6,7}$ \\ Alexander C.H. Geurts MD PhD ${ }^{1,2}$
}

\begin{abstract}
${ }^{1}$ Department of Rehabilitation, Donders Institute for Brain, Cognition and Behaviour, Radboud University Medical Center, Nijmegen, The Netherlands;

${ }^{2}$ Department of Rehabilitation, Sint Maartenskliniek, Nijmegen, The Netherlands

${ }^{3}$ Research Department, Sint Maartenskliniek, Nijmegen, The Netherlands

${ }^{4}$ Department of Orthopaedics, Sint Maartenskliniek, Nijmegen, The Netherlands

${ }^{5}$ Rijndam Rehabilitation Center, Rotterdam, The Netherlands

${ }^{6}$ Roessingh Research and Development, Enschede, the Netherlands

${ }^{7}$ Biomedical Signals and Systems, MIRA - Institute for Biomedical Technology and Technical Medicine, University of Twente, Enschede, The Netherlands
\end{abstract}

Running title: Gait impairments in supratentorial upper motor neuron syndromes

Word count: 3497

\section{Corresponding author}

Jorik Nonnekes, MD, PhD

Radboud University Medical Centre

Department of Rehabilitation

PO Box 19101, 6500 HB Nijmegen

The Netherlands

E-mail: jorik.nonnekes@radboudumc.nl 


\section{ABSTRACT}

Importance: A variety of neurological disorders can damage the corticospinal tract in the supratentorial region of the brain. Gait impairments are common in patients with chronic supratentorial upper motor neuron lesions, and are a source of great disability.

Clinical management aimed at improving the gait pattern in these patients is generally perceived as a challenging task, as many possible abnormalities may interact. Moreover, a multitude of treatment options exist - ranging from assistive devices and muscle stretching to pharmacological and surgical interventions - but evidence is inconclusive for most approaches and there is a lack of clear treatment guidelines.

Observations: To improve management, we here show that gait deviations in adults with a chronic supratentorial upper motor neuron lesion can roughly be reduced to three groups of primary deficits: (1) imbalance of muscle strength, length and activity around the ankle and tarsal joints leading to pes equino(varus), (2) calf muscle weakness, and (3) overactivity of proximal leg muscles.

Conclusions and relevance: We introduce a step-wise treatment algorithm with emphasis on medical-technical interventions, which is based on evidence when available, and otherwise reflects practice-based experience by the authors. 


\section{INTRODUCTION}

A variety of neurological disorders, such as stroke, traumatic brain injury and cerebral tumours, can damage the corticospinal tract in the supratentorial region of the brain. Damage to these descending motor pathways results in a characteristic set of signs and symptoms, which are collectively termed the 'upper motor neuron (UMN) syndrome'. These signs include contralateral loss of muscle force and motor selectivity, exaggerated deep tendon reflexes, and increased muscle tone. In second instance contractures can emerge. ${ }^{1}$ The UMN syndrome commonly results in gait impairments that have a disabling impact on the quality of life of affected individuals. ${ }^{2-4}$

Clinical management aimed at improving the gait pattern in patients with chronic unilateral UMN syndrome is challenging for two main reasons. First, the spastic gait pattern is characterized by many possible abnormalities including - but not limited to - foot drop, reduced knee flexion and circumduction during the swing phase, and abnormal foot landing and lack of stability during the stance phase (see video $1 \mathrm{a}$ and $2 \mathrm{2}$ ). ${ }^{3}$ These gait abnormalities constitute a combination of primary deficits and secondary (sometimes compensatory) mechanisms that can be hard to disentangle. ${ }^{5}$ Second, a multitude of treatment options exist, but clinical evidence is inconclusive for most approaches. As a result, variation in clinical practice is large, indicating the need for clear treatment algorithms. ${ }^{6} \mathrm{~A}$ particular problem in this respect is the underuse of surgical treatment options, even though in some cases the best long-term functional results may be obtained by surgical interventions. ${ }^{6-8}$

This review aims to fill in the lack of a clinical treatment guideline. We will first argue that gait impairments in adults with chronic unilateral UMN syndrome can roughly be reduced to three groups of primary deficits: (1) imbalance of muscle strength, length and activity around the ankle and tarsal joints leading to pes equino(varus), (2) calf muscle weakness, and (3) overactivity of proximal leg muscles. We then provide a stepwise treatment algorithm which is based on evidence when available, and otherwise reflects practice-based experience of the authors.

\section{UNRAVELLING PRIMARY AND SECONDARY GAIT IMPAIRMENTS}

Walking involves the repetition of a regular pattern of limb and trunk movements - collectively termed the gait cycle (see box 1). It is the result of a complex interaction between postural control and leg motor control, and both can be hampered by a unilateral UMN lesion. From studies in patients with stroke, it has become evident that postural control often substantially improves in the subacute phase and that it can be trained to some extent in both the subacute and chronic phases post stroke. ${ }^{9,10}$ In contrast, leg motor control often shows less spontaneous recovery in the subacute 
phase post stroke, and there is only very limited evidence that intensive training can restore leg muscle coordination during gait. ${ }^{11-13}$ As a consequence, many patients with chronic unilateral UMN syndrome show gait abnormalities and asymmetry that prohibit an efficient and stable gait pattern and do not significantly respond to exercise-based interventions. ${ }^{14}$

\section{Altered neural control of walking}

To distinguish between primary gait deficits and secondary mechanisms, knowledge from neuroanatomical and neurophysiological studies is critical. Importantly, these studies have predominantly focused on the upper limb, ${ }^{15,16}$ but findings can reasonably be translated to the lower extremity. Besides the corticospinal tract, there are parallel descending motor pathways originating from the brainstem (e.g. the reticulospinal tract) that can influence trunk and leg motor control. However, only the corticospinal tract is able to produce refined movements, which is especially true for the distal musculature. ${ }^{17,18} \mathrm{~A} U \mathrm{UN}$ lesion will, therefore, have a greater impact on distal than on proximal leg muscles, which implies that movements at the ankle and foot joints are hampered more than movements at the hip joint. Consequently, proximal leg muscles are often used to compensate for distal motor control deficits. In this perspective, pathways descending from the brainstem may compensate for lack of corticospinal motor control. There is emerging evidence that after a supratentorial UMN lesion, there is some degree of neuroplasticity through the cortico-reticulospinal system, thereby bypassing the dysfunctional corticospinal tract. ${ }^{16,19,20}$ Given the cortico-reticular innervation pattern, strengthening of (contralesional) cortico-reticular output will predominantly result in a larger output to leg extensors, and to a lesser degree to leg flexors. Indeed, after corticospinal lesions a 'leg extensor bias' is observed: the recovery of muscle strength is better in the leg extensors (e.g. triceps surae and quadriceps) than in the leg flexors (e.g. tibialis anterior and hamstrings). ${ }^{21}$ It is important to realize, however, that the (partial) recovery of muscle strength of the leg extensors often comes at the price of a higher muscle tone and reflexes ('spasticity') in these muscle groups. ${ }^{22}$

\section{Three primary deficits}

Based on the aforementioned insights as well as on clinical studies and experience, we argue that the gait impairments observed in patients with chronic unilateral UMN syndrome can roughly be reduced to three groups of 'primary' deficits: (1) imbalance of muscle strength, length and activity around the ankle and tarsal joints leading to pes equino(varus), (2) calf muscle weakness, and (3) overactivity of proximal leg muscles. The main impairment is muscular imbalance around the ankle and tarsal joints, resulting in pes equinus or pes equinovarus. ${ }^{23}$ The dorsiflexors and evertors of the foot are usually weak, which can be aggravated by spasticity and contractures of the plantarflexors 
and invertors of the foot. Depending on the degree of spasticity and/or contracture, pes equino(varus) can be 'fixed' or 'dynamic'. Shortening of the triceps surae will result in fixed pes equinus, whereas (additional) shortening of the tibialis posterior will result in fixed pes equinovarus. Mere spastic overactivity of the triceps surae, usually combined with relative weakness of the foot elevators, will lead to dynamic pes equinus. Notably, muscle imbalance between the medial foot elevators (relative good strength of the tibialis anterior) and lateral foot elevators (weakness of the toe extensors and peroneus tertius) may also result in a dynamic pes varus, often with a less pronounced equinus component. ${ }^{24}$

The three 'primary' deficits frequently result in secondary (sometimes compensatory) gait abnormalities (see box 2 ). Hence, adequate treatment of these primary deficits will most likely resolve these secondary mechanisms as well.

\section{ASSESSMENT OF GAIT IMPAIRMENTS}

Assessment of gait in patients with unilateral UMN syndrome starts with history taking, followed by an observation of the gait pattern and clinical examination. History taking is especially important to evaluate the impact of gait impairments on daily life. To properly observe the gait pattern, a patient needs to be able to walk along a trajectory of approximately ten metres, both with and without footwear/orthosis. If possible, a video system should be used to be able to review the gait pattern offline and in slow motion. If available, instrumented gait analysis including kinematic, kinetic, and electromyographic assessment, can aid in discriminating primary from secondary gait abnormalities. ${ }^{6}$ In this perspective, it is important to realize that the presence or absence of 'spasticity' observed during clinical examination often does not translate to muscle (over)activity observed by instrumented analysis of the gait cycle. Dynamic electromyography is, therefore, indispensable to detect or confirm troublesome muscular overactivity during gait, ${ }^{6}$ for example to identify overactivity of calf musculature underlying knee hyperextension during the stance phase or to identify a spastic rectus femoris underlying reduced knee flexion during the swing phase (see box 2).

\section{CLINICAL MANAGEMENT OF GAIT IMPAIRMENTS}

After thorough assessment of the primary deficits contributing to the gait abnormalities in patients with a chronic supratentorial UMN lesion, treatment focuses on improvement of these primary deficits (gait pattern), with the ultimate goal to improve daily-life walking capacity, for example, to walk safely without aids or to walk longer distances. It is important that such specific performance goals are set by shared decision-making. In the remainder of this text, we will elaborate on the treatment options for each of these deficits, with emphasis on medical-technical interventions. 


\section{Pes equino(varus)}

As described above, pes equino(varus) is the result of muscular imbalance around the ankle and tarsal joints, where relative weakness of the anterolateral lower leg muscles is often accompanied by spasticity and shortening of the medioposterior lower leg muscles. Here, we take two important considerations into account. First, because the mechanical or electrical support of weakened muscles is not effective when antagonist muscles produce counteractive forces, we propose a hierarchical approach to the treatment of pes equino(varus), starting with the treatment of contracture, followed by the treatment of spasticity, and ending by the provision of support to weakened muscles. A second consideration relates to the distinction between pes equinus and pes equinovarus. For an adequate selection of treatment options, it is of critical importance to identify whether there is any varus deformity of the hindfoot at initial contact or during the stance phase that predisposes to instability while loading. If so, treatment of varus deformity is essential to restore the main prerequisite of gait: stability in the stance phase. Because of this importance, and because treatment of varus deformity requires specific interventions, our algorithm consists of two interrelated flow diagrams: one for pes equinovarus (left side) and one for pes equinus (right side), see figure 1.

In case of a relevant contracture contributing to fixed pes equino(varus), serial casting or stretching exercises should be considered as a first step, although this treatment option is more likely to be successful in relative young patients and if the intended gain in passive ankle dorsiflexion does not exceed $10-15^{\circ}$. If concomitant spasticity of the plantarflexors and invertors hampers adequate muscle stretch, supportive neuromuscular blockade (e.g. with botulinum toxin) should be considered. Typical target muscles are the medial and lateral gastrocnemius (in case of fixed equinus deformity with stretched knee), soleus (in case of fixed equinus deformity with flexed knee), tibialis posterior (in case of fixed varus deformity), and toe flexors (in case of fixed flexion deformity of toes). The combination of serial casting for one week preceded by neuromuscular blockade of the calf muscles has shown to improve performance on the 10-meter walk test and 6-minute walk test, whereas the combination of a stretching regime with neuromuscular blockade of the calf muscles did not yield significant results. ${ }^{25}$ However, both intensive stretching and serial casting may be troublesome or lead to disappointing results in many patients. In these cases, a surgical intervention should be considered. If both the gastrocnemius and soleus muscles are shortened, Achilles tendon lengthening is indicated. Instead, if there is only equinus deformity with a stretched knee, a fasciotomy of the gastrocnemius muscles may be sufficient. In case of additional fixed varus deformity during stance or toe flexion deformity, the above-mentioned procedures can be combined with an intramuscular tenotomy or transfer of the tibialis posterior muscle and toe flexors, 
respectively. In case of varus deformity, fusion of one or more tarsal joints can also be considered to obtain optimal stability of the foot in the frontal plane (see video $1 \mathrm{~b}$ ). In the presence of toe flexion deformity, additional fixation of the interphalangeal toe joints should be considered as well. These surgical interventions often remain underused, even though the inability to easily achieve a plantigrade foot position with extended toes while loading the leg often causes huge functional problems. ${ }^{6}$ As shown in table 1 , the formal evidence for surgical interventions to correct pes equinovarus is still sparse due to the absence of well controlled studies, ${ }^{7}$ but clinical experience is extensive and consistently positive if surgery is indicated and performed by an experienced and dedicated team. An important advantage of lengthening procedures of muscles is that concomitant spasticity is also markedly and more or less permanently reduced after surgery, perhaps due to a shift in the mechanical threshold at which spasticity is triggered. ${ }^{26}$ When a surgical intervention is contraindicated, orthopaedic footwear should be considered to stabilize the structural ankle-foot deformity, restore the base of support, and provide some form of roll-off correction.

After treatment of relevant contractures, or in the absence of contractures, relevant (residual) spasticity contributing to dynamic pes equino(varus) should be treated. Focal spasmolysis is preferred to systemic treatment in order to improve the gait pattern. For treating spastic pes equinovarus, typical target muscles are the medial and lateral gastrocnemius, soleus, tibialis posterior, and toe flexors. Focal spasmolysis can be performed by neuromuscular blockade (e.g. with botulinum toxin) or by a block of the tibial nerve (e.g. with phenol). A relatively small, double-blind randomized controlled trial (RCT) in 20 patients with spastic equinovarus compared intramuscular injections with botulinum toxin type $A$ to tibial nerve blockade with phenol. Both treatment modalities reduced the time needed to walk 25 feet, but the improvements were only significant in the group treated with botulinum toxin. ${ }^{27}$ Based on the absence of sensory side effects such as sensibility loss or neuralgia, neuromuscular blockade is preferred to nerve block by many clinicians, although some have the impression that nerve block may be more effective to silence severe ankle clonus. A systematic review and meta-analysis showed that treatment of spastic pes equinovarus with botulinum toxin type $A$ is associated with a small but significant improvement in gait velocity $(0.044 \mathrm{~m} / \mathrm{s}) .^{28}$ The clinical importance of this improvement in gait speed can be debated, but it is our experience that focal spasmolyis of the calf muscles can have a relevant beneficial effect on the gait pattern by reducing an excessive ankle plantarflexion - knee extension couple.

If focal spasmolytic treatment of the dorsal lower leg muscles is unavailable, insufficient or short lasting, a more permanent improvement can be obtained by subsequent surgical intervention. Selective neurotomy of tibial nerve branches may be performed, based on the temporary effects of precision blocks of the tibial nerve with a local anaesthetic. ${ }^{29} \mathrm{~A}$ small RCT in 16 patients with spastic 
pes equinovarus compared selective neurotomy of the soleus, tibialis posterior, and flexor hallucis longus to intramuscular injections with botulinum toxin type $A$ and found equal improvement of ankle kinematics with preservation of ankle kinetics during gait, but more reduction of ankle stiffness after neurotomy. ${ }^{30}$ Alternatively, for elimination of the dynamic varus component and/or dynamic toe flexor deformity due to spasticity, an intramuscular tenotomy or transfer of the tibialis posterior muscle and/or toe flexors should be considered. In these cases, even selective forms of arthrodesis (of the tarsal and/or interphalangeal toe joints) can be indicated to obtain optimal long-term effects, depending on the severity of spasticity.

After optimal treatment of relevant contractures and spasticity, or in the absence of both, any (residual) dynamic pes equino(varus) will be the result of muscle weakness. In these cases, muscle weakness is usually supported by some type of ankle-foot orthosis (AFO) or by functional electrical stimulation (FES) of the common peroneal nerve. The main disadvantage of these interventions is that they cannot be applied 24-hours a day, and particularly AFOs may limit other activities such as kneeling, ascending and descending stairs, cycling or car driving. For this reason, any orthotic intervention should support loss of ankle-foot function as dynamically as possible. To minimize the necessity for orthotic interference, surgical correction of the varus component should be considered, in particular in patients who value barefoot walking. Because a varus deviation of the foot during the loading response in patients without relevant contracture or spasticity is often caused by an imbalance among the foot dorsiflexors in favour of the tibialis anterior muscle, a split anterior tibial tendon transfer (SPLATT; see video $2 \mathrm{~b}$ ) is an option if the tibialis anterior shows sufficient strength (close to normal) and motor selectivity (ability to selectively dorsiflex the foot with an extended knee). In this procedure, a lateral part of the tibialis anterior tendon is transferred to the cuboid or peroneus tertius tendon to rebalance the foot during dorsiflexion. Although the effect of a SPLATT alone has not been investigated, a combined Achilles tendon lengthening and SPLATT procedure resulted in a spatiotemporal normalization of the single-stance and double-support phases of gait. ${ }^{31}$ If the tibialis anterior muscle is too weak to perform a SPLATT, there is still the possibility of a fusion of one or more tarsal joints. This entails the advantage of far less instability of the hindfoot during loading when walking barefoot. In addition, after this surgical procedure, an AFO can be strictly focused on the dynamic support of foot dorsiflexion without the need of providing static stability in the frontal plane. Dynamic pes equinus due to muscle weakness requires either mechanical or electrical support of the foot dorsiflexion during the swing phase and loading phase. A systematic and pooled meta-analysis showed that an AFO significantly improves several aspects of walking, including gait speed, loading symmetry, and the ability to walk independently. ${ }^{32}$ In case of excessive knee flexion during the stance phase, a rigid AFO with a ventral shell should be considered to 
optimize both foot clearance during the swing phase and knee control during the stance phase. In case of excessive knee extension during the stance phase, a rigid AFO with a dorsal shell should be considered for the same reasons. With fair to good knee control, peroneal FES is a good alternative to AFO. The main theoretical advantages of FES over AFO are optimal passive ankle motion during roll-off and optimal preservation of residual push-off power and related swing-phase initiation. Surface (external) FES has shown to be equivalent to AFO in terms of gait speed and functional gait tests in several RCTs. ${ }^{33,34}$ Some studies of implanted peroneal FES systems have shown indications of an improved gait pattern and gait symmetry as well. ${ }^{35-37}$

\section{Calf muscle weakness}

Unfortunately, there is currently no treatment option that effectively compensates for calf muscle weakness. There are some indications that it is possible to improve the propulsion symmetry between the legs in patients with stroke through targeted training, either by increasing gait speed with or without $\mathrm{FES}^{38,39}$ or by walking on a treadmill with body weight support. ${ }^{40}$ However, there are large variations in training responses between subjects and more research needs to be done to identify 'responders' and establish long-term effects of such training regimens. In the near future, studies investigating energy-storing ankle foot orthosis or next generation (light-weight, soft-suit) exoskeletons may be able to prove that push-off power can effectively be mechanically supported, leading to a better swing-phase initiation. Exoskeletons may also be able to support hip flexion power in patients with combined weakness of the calf muscles and hip flexors.

\section{Overactivity of proximal leg muscles}

In the presence of specific overactivity of the rectus femoris during early swing, a focal treatment option is preferred, either by neuromuscular blockade or by a block of the motor branch of the femoral nerve supplying the rectus femoris muscle. A systematic review reported that both neuromuscular blockade and motor branch block resulted in a higher peak knee flexion angle and velocity during gait, but improvement in walking speed was not consistently observed. ${ }^{41}$ Both treatment modalities were unable to restore normal knee flexion, which can be explained by the notion that part of the 'stiff knee' problem in these patients may be due to concomitant calf muscle weakness limiting push-off power. ${ }^{42}$ An alternative treatment to neuromuscular blockade or nerve block is surgical rectus femoris transfer. ${ }^{43}$ In this procedure, the distal part of the rectus femoris muscle is transferred to the gracilis muscle with concomitant fractional lengthening of the vastus muscles. Yet, very few scientific data on this procedure are available.

When there is general overactivity of the thigh muscles that interferes with the gait pattern, the use of oral spasmolytic drugs may be considered. A significant improvement on walking distance has 
been reported for tizanidine ${ }^{44}$ and tolperisone ${ }^{45}$ in patients with stroke. However, oral spasmolytic drugs may easily cause systemic side effects (e.g. drowsiness, nausea) and are usually ineffective to obtain specific gait improvements. ${ }^{26}$ When the use of oral drugs is limited due to side effects, intrathecal baclofen (ITB) administration is an alternative systemic treatment option to improve gait speed and distance in patients with stroke ${ }^{46}$ and reduce the extensor co-activation of rectus femoris and gastrocnemius in patients with acquired brain injury. ${ }^{47}$

\section{CONCLUSIONS AND FUTURE DIRECTIONS}

The management of gait impairments in patients with chronic supratentorial UMN syndrome is complex from both a diagnostic and therapeutic point of view. Our step-wise approach to the management of these gait impairments is a first attempt to fill in the lack of a clinical treatment guideline, while at the same time acknowledging that many more evidence needs to be obtained by sound comparative studies. As such, table 1 may serve as a template for future studies. Importantly, there is need for trials that do not investigate the effect of only one intervention, but rather the combined effect of several (subsequent) treatment options, as presented in our algorithm. Outcomes should include the attainment of personal goals (e.g. the ability to walk without a walking aid, or to walking for longer distances), consider barefoot walking in addition to walking with shoes, and take the effects on non-walking daily-life activities into account as well. Although we purposely focused on medical-technical interventions to improve the gait pattern (and not on exercise-based interventions), it should be mentioned that many of the interventions addressed in this paper may still profit from additional functional gait training.

\section{Contributors}

JN and NB did the literature search. All authors were involved in drafting of the manuscript and design of the flowchart. 


\section{References}

1. Gracies JM. Pathophysiology of spastic paresis. I: Paresis and soft tissue changes. Muscle Nerve. 2005;31(5):535-551.

2. Weerdesteyn V, de Niet M, van Duijnhoven HJ, Geurts AC. Falls in individuals with stroke. Journal of rehabilitation research and development. 2008;45(8):1195-1213.

3. Snijders AH, van de Warrenburg BP, Giladi N, Bloem BR. Neurological gait disorders in elderly people: clinical approach and classification. The Lancet Neurology. 2007;6(1):63-74.

4. Hyndman D, Ashburn A, Stack E. Fall events among people with stroke living in the community: Circumstances of falls and characteristics of fallers. Archives of physical medicine and rehabilitation. 2002;83(2):165-170.

5. Schmid S, Schweizer K, Romkes J, Lorenzetti S, Brunner R. Secondary gait deviations in patients with and without neurological involvement: A systematic review. Gait \& posture. 2013;37(4):480-493.

6. Patrick JH, Keenan MA. Gait analysis to assist walking after stroke. Lancet. 2007;369(9558):256-257.

7. Renzenbrink GJ, Buurke JH, Nene AV, Geurts AC, Kwakkel G, Rietman JS. Improving walking capacity by surgical correction of equinovarus foot deformity in adult patients with stroke or traumatic brain injury: a systematic review. Journal of rehabilitation medicine. 2012;44(8):614-623.

8. Reddy S, Kusuma S, Hosalkar H, Keenan MA. Surgery can reduce the nonoperative care associated with an equinovarus foot deformity. Clinical orthopaedics and related research. 2008;466(7):1683-1687.

9. Vearrier LA, Langan J, Shumway-Cook A, Woollacott M. An intensive massed practice approach to retraining balance post-stroke. Gait Posture. 2005;22(2):154-163.

10. Marigold DS, Eng JJ, Dawson AS, Inglis JT, Harris JE, Gylfadottir S. Exercise leads to faster postural reflexes, improved balance and mobility, and fewer falls in older persons with chronic stroke. Journal of the American Geriatrics Society. 2005;53(3):416-423.

11. Routson RL, Clark DJ, Bowden MG, Kautz SA, Neptune RR. The influence of locomotor rehabilitation on module quality and post-stroke hemiparetic walking performance. Gait \& posture. 2013;38(3):511-517.

12. Buurke JH, Nene AV, Kwakkel G, Erren-Wolters V, IJzerman MJ, Hermens HJ. Recovery of Gait After Stroke: What Changes? Neurorehabilitation and neural repair. 2008;22(6):676-683.

13. Den Otter AR, Geurts ACH, Mulder T, Duysens J. Gait recovery is not associated with changes in the temporal patterning of muscle activity during treadmill walking in patients with poststroke hemiparesis. Clinical Neurophysiology. 2006;117(1):4-15.

14. Harris JE, Eng JJ, Marigold DS, Tokuno CD, Louis CL. Relationship of balance and mobility to fall incidence in people with chronic stroke. Phys Ther. 2005;85(2):150-158.

15. Davidson AG, Buford JA. Bilateral actions of the reticulospinal tract on arm and shoulder muscles in the monkey: stimulus triggered averaging. Exp Brain Res. 2006;173(1):25-39.

16. Baker SN. The primate reticulospinal tract, hand function and functional recovery. J Physiol. 2011;589(Pt 23):5603-5612.

17. Lawrence DG, Kuypers HG. The functional organization of the motor system in the monkey. I. The effects of bilateral pyramidal lesions. Brain. 1968;91(1):1-14.

18. Lawrence DG, Kuypers HG. The functional organization of the motor system in the monkey. II. The effects of lesions of the descending brain-stem pathways. Brain. 1968;91(1):15-36.

19. Nonnekes J, Oude Nijhuis LB, de Niet M, et al. StartReact restores reaction time in HSP: evidence for subcortical release of a motor program. J Neurosci. 2014;34(1):275-281.

20. Jang SH, Chang CH, Lee J, Kim CS, Seo JP, Yeo SS. Functional Role of the Corticoreticular Pathway in Chronic Stroke Patients. Stroke. 2013;44(4):1099-1104.

21. Sheffler LR, Chae J. Hemiparetic Gait. Phys Med Rehabil Clin N Am. 2015;26(4):611-623.

22. Li S, Francisco GE. New insights into the pathophysiology of post-stroke spasticity. Front Hum Neurosci. 2015;9.

23. Keenan MA, Creighton J, Garland DE, Moore T. Surgical correction of spastic equinovarus deformity in the adult head trauma patient. Foot \& ankle. 1984;5(1):35-41.

24. Perry J, Waters RL, Perrin T. Electromyographic analysis of equinovarus following stroke. Clinical orthopaedics and related research. 1978(131):47-53. 
25. Carda S, Invernizzi M, Baricich A, Cisari C. Casting, taping or stretching after botulinum toxin type A for spastic equinus foot: a single-blind randomized trial on adult stroke patients. Clin Rehabil. 2011;25(12):1119-1127.

26. Guissard N, Duchateau J. Neural aspects of muscle stretching. Exerc Sport Sci Rev. 2006;34(4):154-158.

27. Kirazli Y, On AY, Kismali B, Aksit R. Comparison of phenol block and botulinus toxin type A in the treatment of spastic foot after stroke: a randomized, double-blind trial. American journal of physical medicine \& rehabilitation. 1998;77(6):510-515.

28. Foley N, Murie-Fernandez M, Speechley M, Salter K, Sequeira K, Teasell R. Does the treatment of spastic equinovarus deformity following stroke with botulinum toxin increase gait velocity? A systematic review and meta-analysis. European journal of neurology. 2010;17(12):1419-1427.

29. Deltombe T, Wautier D, De Cloedt P, Fostier M, Gustin T. Assessment and treatment of spastic equinovarus foot after stroke: Guidance from the Mont-Godinne interdisciplinary group. Journal of rehabilitation medicine. 2017;49(6):461-468.

30. Bollens B, Gustin T, Stoquart G, Detrembleur C, Lejeune T, Deltombe T. A randomized controlled trial of selective neurotomy versus botulinum toxin for spastic equinovarus foot after stroke. Neurorehabilitation and neural repair. 2013;27(8):695-703.

31. Pinzur MS, Sherman R, Dimontelevine P, Kett N, Trimble J. Adult-Onset Hemiplegia - Changes in Gait after Muscle-Balancing Procedures to Correct the Equinus Deformity. J Bone Joint Surg Am. 1986;68A(8):1249-1257.

32. Tyson SF, Kent RM. Effects of an ankle-foot orthosis on balance and walking after stroke: a systematic review and pooled meta-analysis. Archives of physical medicine and rehabilitation. 2013;94(7):1377-1385.

33. Bethoux F, Rogers HL, Nolan KJ, et al. The effects of peroneal nerve functional electrical stimulation versus ankle-foot orthosis in patients with chronic stroke: a randomized controlled trial. Neurorehabilitation and neural repair. 2014;28(7):688-697.

34. Kluding PM, Dunning K, O'Dell MW, et al. Foot drop stimulation versus ankle foot orthosis after stroke: 30-week outcomes. Stroke. 2013;44(6):1660-1669.

35. Howlett OA, Lannin NA, Ada L, McKinstry C. Functional electrical stimulation improves activity after stroke: a systematic review with meta-analysis. Archives of physical medicine and rehabilitation. 2015;96(5):934-943.

36. Kottink Al, Hermens HJ, Nene AV, Tenniglo MJ, Groothuis-Oudshoorn CG, MJ IJ. Therapeutic effect of an implantable peroneal nerve stimulator in subjects with chronic stroke and footdrop: a randomized controlled trial. Physical therapy. 2008;88(4):437-448.

37. Schiemanck S, Berenpas F, van Swigchem R, et al. Effects of implantable peroneal nerve stimulation on gait quality, energy expenditure, participation and user satisfaction in patients with post-stroke drop foot using an ankle-foot orthosis. Restorative neurology and neuroscience. 2015;33(6):795-807.

38. Beaman CB, Peterson CL, Neptune RR, Kautz SA. Differences in self-selected and fastestcomfortable walking in post-stroke hemiparetic persons. Gait \& posture. 2010;31(3):311-316.

39. Awad LN, Reisman DS, Kesar TM, Binder-Macleod SA. Targeting paretic propulsion to improve poststroke walking function: a preliminary study. Archives of physical medicine and rehabilitation. 2014;95(5):840-848.

40. Bowden MG, Behrman AL, Neptune RR, Gregory CM, Kautz SA. Locomotor rehabilitation of individuals with chronic stroke: difference between responders and nonresponders. Archives of physical medicine and rehabilitation. 2013;94(5):856-862.

41. Tenniglo MJ, Nederhand MJ, Prinsen EC, Nene AV, Rietman JS, Buurke JH. Effect of chemodenervation of the rectus femoris muscle in adults with a stiff knee gait due to spastic paresis: a systematic review with a meta-analysis in patients with stroke. Archives of physical medicine and rehabilitation. 2014;95(3):576-587.

42. Kerrigan DC, Karvosky ME, Riley PO. Spastic paretic stiff-legged gait - Joint kinetics. American journal of physical medicine \& rehabilitation. 2001;80(4):244-249.

43. Namdari S, Pill SG, Makani A, Keenan MA. Rectus femoris to gracilis muscle transfer with fractional lengthening of the vastus muscles: a treatment for adults with stiff knee gait. Physical therapy. 2010;90(2):261-268. 
44. Bes A, Eyssette M, Pierrot-Deseilligny E, Rohmer F, Warter JM. A multi-centre, double-blind trial of tizanidine, a new antispastic agent, in spasticity associated with hemiplegia. Current medical research and opinion. 1988;10(10):709-718.

45. Stamenova P, Koytchev R, Kuhn K, et al. A randomized, double-blind, placebo-controlled study of the efficacy and safety of tolperisone in spasticity following cerebral stroke. European journal of neurology. 2005;12(6):453-461.

46. Schiess MC, Oh IJ, Stimming EF, et al. Prospective 12-month study of intrathecal baclofen therapy for poststroke spastic upper and lower extremity motor control and functional improvement. Neuromodulation : journal of the International Neuromodulation Society. 2011;14(1):38-45; discussion 45.

47. Chow JW, Yablon SA, Stokic DS. Intrathecal baclofen bolus reduces exaggerated extensor coactivation during pre-swing and early-swing of gait after acquired brain injury. Clin Neurophysiol. 2017;128(5):725-733.

48. Kaji R, Osako $Y$, Suyama $K$, et al. Botulinum toxin type $A$ in post-stroke lower limb spasticity: a multicenter, double-blind, placebo-controlled trial. Journal of neurology. 2010;257(8):13301337.

49. Ferreira LA, Neto HP, Grecco LA, et al. Effect of Ankle-foot Orthosis on Gait Velocity and Cadence of Stroke Patients: A Systematic Review. Journal of physical therapy science. 2013;25(11):1503-1508.

50. Kottink Al, Oostendorp L, Buurke JH, Nene AV, Hermens HJ, MJ IJ. The orthotic effect of functional electrical stimulation on the improvement of walking in stroke patients with a dropped foot: a systematic review. Artificial organs. 2004;28(6):577-586.

51. Kottink Al, Hermens HJ, Nene AV, et al. A randomized controlled trial of an implantable 2channel peroneal nerve stimulator on walking speed and activity in poststroke hemiplegia. Archives of physical medicine and rehabilitation. 2007;88(8):971-978.

52. Bensoussan L, Mathelin A, Viton JM, Collado H, Delarque A. Improvement of gait in a stroke patient. A 7-year longitudinal study. Disability and rehabilitation. 2010;32(20):1705-1711.

53. J. Perry JMB. Gait Analysis, Normal and Pathological Function, 2nd Burnfield, Slack Inc, ISBN 978-1-55642r-r766-4.

54. Chen G, Patten C, Kothari DH, Zajac FE. Gait differences between individuals with post-stroke hemiparesis and non-disabled controls at matched speeds. Gait \& posture. 2005;22(1):51-56.

55. Winter DA. The biomechanics and motor control of human gait: Normal eapneWOUoWP. The biomechanics and motor control of human gait: Normal, elderly and pathological. . 1991;2nd ed. Waterloo (Ontario): University of Waterloo Press.

56. Goldberg SR, Anderson FC, Pandy MG, Delp SL. Muscles that influence knee flexion velocity in double support: implications for stiff-knee gait. J Biomech. 2004;37(8):1189-1196. 
Table 1: level of evidence of interventions for pes equino(varus) in patients with chronic supratentorial upper motor neuron syndrome

\begin{tabular}{|c|c|c|}
\hline Intervention & Effect & $\begin{array}{l}\text { Level of } \\
\text { evidence* }\end{array}$ \\
\hline \multicolumn{3}{|c|}{ Management of pes equino(varus) due to contractures } \\
\hline Stretching & Improvement in case of mild pes equines & $\mathrm{D}$ \\
\hline $\begin{array}{l}\text { Stretching after botulinum toxin } \\
\text { injection in calf musculature }\end{array}$ & No significant improvement & $B^{25}$ \\
\hline $\begin{array}{l}\text { Serial casting after botulinum toxin } \\
\text { injection in calf musculature }\end{array}$ & Improvement in gait speed & $B^{25}$ \\
\hline Achilles tendon lengthening & Improvement & $\mathrm{D}$ \\
\hline Fasciotomy of gastrocnemius & Improvement & $\mathrm{D}$ \\
\hline Tenotomy of tibials posterior & Improvement & $\mathrm{D}$ \\
\hline \multicolumn{3}{|c|}{ Management of pes equino(varus) due to spasticity } \\
\hline Botulinum toxin in calf musculature & Tendency towards small improvement in gait speed & $\mathrm{A} 2^{28,48}$ \\
\hline $\begin{array}{l}\text { Chemodenervation of tibial nerve } \\
\text { using phenol }\end{array}$ & Improvement, but no significant effect on gait speed & $\mathrm{B}^{27}$ \\
\hline $\begin{array}{l}\text { Selective peripheral tibial } \\
\text { neurotomy }\end{array}$ & $\begin{array}{l}\text { Improvement in ankle kinematics during gait, no } \\
\text { significant effect on gait speed }\end{array}$ & $\mathrm{B}^{30}$ \\
\hline
\end{tabular}

\section{Management of pes equino(varus) due to muscle weakness}

\begin{tabular}{llc}
\hline $\begin{array}{l}\text { Split anterior tibial tendon transfer } \\
\text { (SPLATT) }\end{array}$ & Improvement & $\mathrm{D}$ \\
\hline Split anterior tibial tendon transfer & Improvement normalization of stance and double & $\mathrm{B}^{31}$ \\
(SPLATT) combined with Achilles & support phases of gait (which had been prolonged & \\
tendon lengthening & before surgery) & $\mathrm{A1}^{32,49}$ \\
\hline Ankle-foot orthosis & Improvement in gait speed, step/stride length and & $\mathrm{A}^{35,50}$ \\
& symmetry of weight distribution & \\
External functional electrical & Improvement in gait speed & $\mathrm{B}^{51}$ \\
stimulation of common peroneal & & \\
nerve & & \\
\hline Implantable functional electrical & Improvement in gait speed and step length symmetry & \\
stimulation of common peroneal & &
\end{tabular}

\begin{tabular}{llc}
\hline Interventions that can target both contractures, spasticity or muscle weakness & \\
\hline Orthopaedic footwear & Improvement in gait speed and gait characteristics such & $\mathrm{B}^{52}$ \\
& as step length and stance duration & \\
\hline Tarsal fusion & Improvement & $\mathrm{D}$ \\
\hline
\end{tabular}


*Level A1: Meta-analysis containing at least some trials of level A2 and of which the results of the trials are consistent; A2: Randomized comparative clinical trials of good quality (randomized double-blind controlled trials) of sufficient size and consistency; B: Randomized clinical trials of moderate (weak) quality of insufficient size or other comparative trials (non-randomized, cohort studies, patient-control studies); C: Non-comparative trials; D: Expert opinion. 


\section{Box 1: The normal gait cycle}

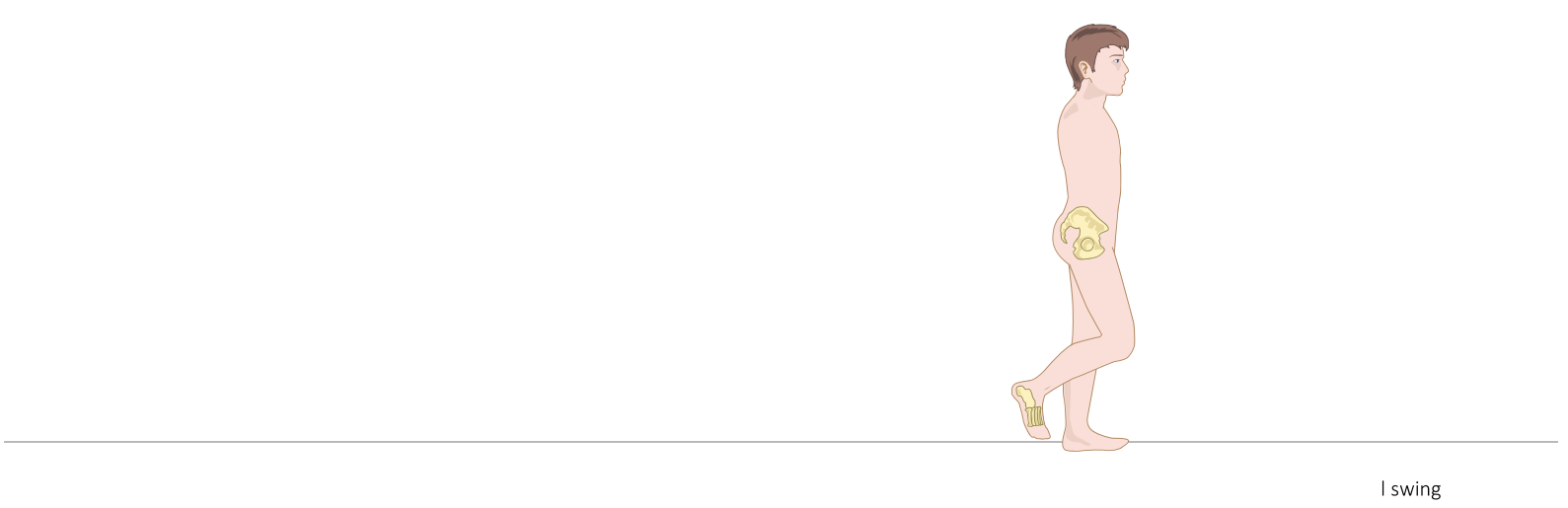

The gait cycle is often subdivided into eight phases, ${ }^{53}$ however, in daily clinical practice, we prefer to separate it into five phases The first phase is the loading response, which starts when the foot strikes the floor (initial contact) and body weight is being transferred to the stance leg. In a normal gait pattern, the heel strikes the floor first, with the ankle in a neutral position. At heel strike, the knee is extended, but during the loading response it flexes for shock absorption, while the ankle shows plantar flexion. The loading response is followed by midstance, which starts when the stance leg has full foot contact and the contralateral leg has started the swing phase. During midstance, the body progresses over the stance leg in a coordinated fashion such that optimal stability and energy conservation are preserved. There is dorsiflexion at the ankle, while the knee and hip extend. Midstance is followed by terminal stance, which starts when the ankle plantarflexes again and the heel rises from the floor, while the knee and hip are still extended. The foot unwinds over the heads of the metatarsal bones and concentric contraction of the calf musculature provides a push-off that generates most of the energy during gait. The last part of terminal stance is characterized by knee and hip flexion, while body weight is gradually transferred to the contralateral side. The fourth phase is early swing, which starts when the foot is lifted from the floor as a result of a coordinated hip and knee flexion and ankle dorsiflexion. Soon thereafter, the knee starts to extend again, while the ankle maintains in a neutral position. The final phase is terminal swing, which starts when the swing leg has passed the contralateral stance leg. In this phase, the hip reaches maximal flexion and the knee full extension to subserve optimal step length and adequate foot positioning for the next loading response. 
Box 2: Primary gait deficits and secondary mechanisms

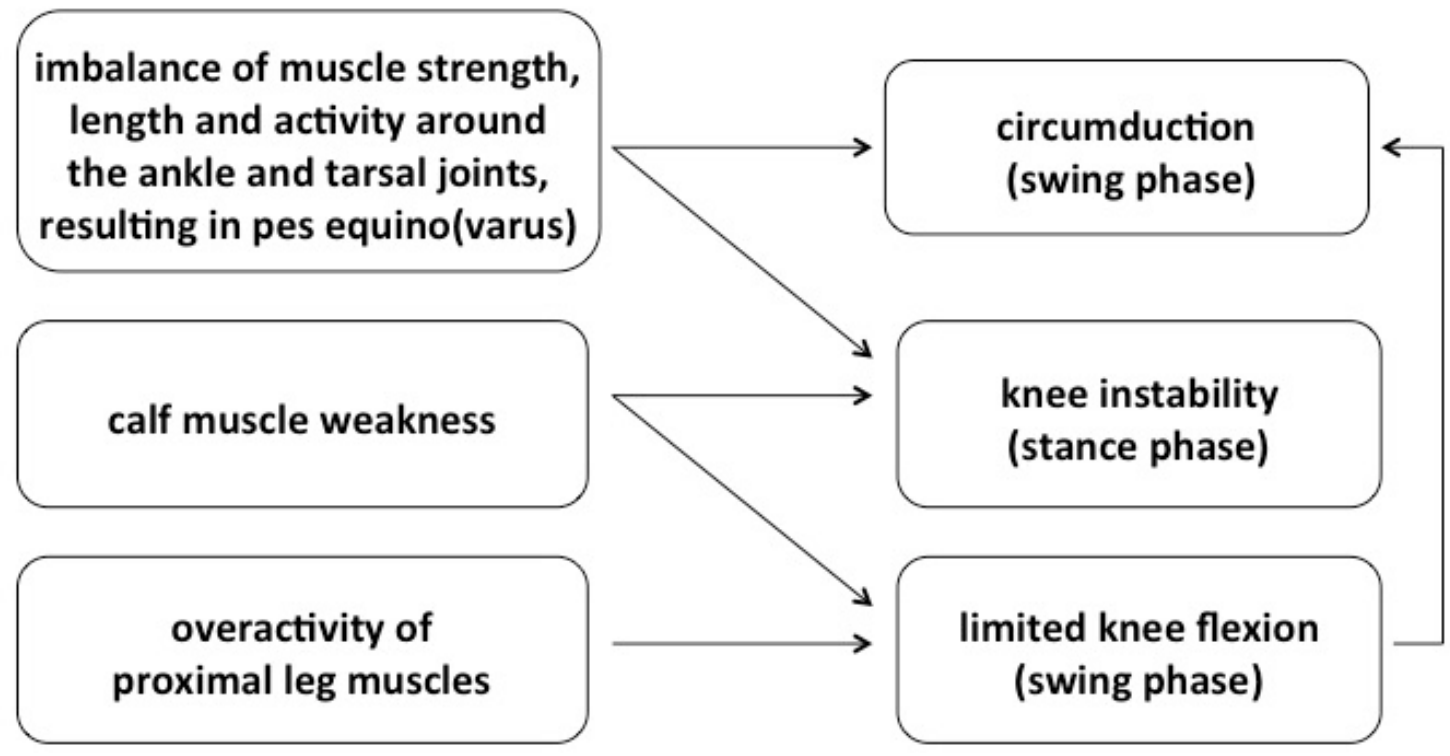

The first 'primary' gait deficit, pes equino(varus), can lead to two important secondary abnormalities at the level of knee and hip-pelvis. First, if equinus deviation occurs during the swing phase, foot clearance is hampered for which a compensatory circumduction movement is needed. ${ }^{54}$ Second, many patients will show knee hyperextension during the stance phase of gait, because a forefoot landing due to pes equinus induces an external knee extension moment. This so-called 'ankle plantarflexion - knee extension' couple hampers energy conservation and impairs a normal roll off of the foot.

The second primary deficit is calf muscle weakness or lack of calf muscle activation. Because the calf is the main stabilizer of the knee joint during midstance, calf muscle weakness can lead to excessive knee flexion in the stance phase. In terminal stance, ankle plantarflexion produces about $75 \%$ of the energy needed for propulsion during normal gait. ${ }^{55} \mathrm{As}$ such, ankle plantarflexion power is the main mechanism underlying knee flexion during the swing phase. ${ }^{56}$ As a consequence, reduced push-off power will often lead to reduced knee flexion during the swing phase, ${ }^{42}$ which may require compensatory circumduction. This situation should be discriminated from the spastic 'stiff knee gait', which is caused by overactive proximal leg extensors.

Overactive proximal leg muscles are the third primary deficit. Especially spasticity of the rectus femoris, hampers further knee flexion ('stiff knee') during the swing phase. ${ }^{41}$ This condition requires even more compensatory circumduction. 
Figure 1: Stepwise treatment algorithm for gait impairments due to pes equino(varus) in patients with unilateral UMN syndrome.

Legend:

* if varus is present in the swing phase, but not during the loading response, this is not considered problematic. **tarsal fusion: one or more tarsal joints (talo-navicular, calcaneo-navicular, calcaneotalar) may be fused, depending on the (expected) destabilizing forces. ${ }^{* *}$ after adequate treatment of varus component. 


\section{Video legends}

\section{Video 1a:}

Gait pattern in a woman, five years after a stroke in the right hemisphere. Stability in the loading and stance phase is severely hampered due to a fixed pes equinovarus deformity.

\section{Video 1b:}

Gait pattern after surgical correction of the pes equinovarus deformity (fasciotomy of the gastrocnemius muscles combined with a talonavicular arthrodesis).

\section{Video 2a:}

Gait pattern in a woman in the chronic phase post stroke. Stability in the loading and stance phase is hampered due to a dynamic pes equinovarus deformity.

\section{Video 2b:}

Gait pattern after surgical procedure involving Achilles tendon lengthening, SPLATT (split anterior tibial tendon transfer), and intramuscular tenotomy of the tibialis posterior muscle and toe flexors. 